\title{
Tasas de logro educativo en las poblaciones afrodescendiente, indígena y mestiza: Revisión de algunos factores de exclusión
}

\section{Rates of Educational Attainment in the Afro-descendant, Indigenous and Mestizo Populations: Review of Some Exclusion Factors}

\author{
Marcelo Gaete-Astica' \\ Ministerio de Educación Pública \\ Departamento de Estudios e Investigación Educativa \\ mgaetea43@gmail.com
}

Recibido 5 de marzo de 2014 • Corregido 28 de setiembre de 2015 - Aceptado 17 de noviembre de 2015

\begin{abstract}
Resumen. Este artículo es resultado de la investigación Tasas de logro educativo entre la población afrodescendiente, indígena y mestiza y la revisión de algunos factores de exclusión e inclusión (Gaete, 2014). Mediante la exploración de los datos del $X$ Censo Nacional de Población y el establecimiento de algunas medidas cuantitativas, en este artículo se busca establecer el logro educativo de la población de personas de origen indígena y afrodescendiente, en contraposición con la población mestiza (que denominamos acríticamente blanca) y algunos elementos como los de la localización provincial y el de inserción temprana en el mundo del trabajo como factores de exclusión educativa. Siendo este estudio de corte exploratorio, se pretende que dé insumos prácticos y conceptuales para profundizar en la temática de la exclusión social y educativa. El análisis estadístico realizado ha permitido visualizar el hecho de que la identidad étnica y cultural, así como la localización geográfica (a escala provincial) no son factores de exclusión educativa y social, sino que las explicaciones hay que buscarlas en la circularidad de la pobreza, que determina logros educativos bajos que influyen, a su vez, el acceso a puestos de trabajo de baja calificación. Este estudio sugiere que la inserción temprana en el mercado de trabajo es un factor clave de exclusión.
\end{abstract}

Palabras claves. Logro educativo, exclusión educativa, factores de exclusión, identidad étnico -cultural, trabajo adolescente.

Abstract. This article is the result of the research Rates of Educational Attainment among the Afro-descendant, Indigenous and Mestizo Populations and the Review of Some Exclusion and Inclusion Factors (Gaete, 2014). This article studies the 10th National Population Census and applies some quantitative measures in an attempt to establish the educational attainment of individuals of aboriginal and Afro-American descent, in contrast to that of the mestizo population (uncritically labeled as "white"). Additionally, educational exclusion factors are explored such as geographical location of the populations of interest and insertion of under-aged population in the job market. Since this study has an exploratory scope, it is intended to give practical and conceptual inputs to delve into the social and educational exclusion theme. Statistical analysis conducted offers a view of the fact that ethnic and cultural identity, as well as geographic location (at a provincial scale) do not seem to be determining factors of educational and social exclusion. On the contrary, explanations must be sought in the circular character of poverty that determines low educational attainment, which in turn determines access to low-level jobs. In this sense, this study suggests that early insertion in the job market is a key exclusionary factor.

Keywords. Educational attainment, educational exclusion, exclusionary factors, ethnic-cultural identity, teenage job market.

${ }^{1}$ Máster en Antropología. Investigador del Departamento de Estudios e Investigación Educativa (DEIE) Ministerio de Educación Pública. Participación en diversas investigaciones del DEIE no publicadas. Costa Rica. 
doi: http://dx.doi.org/10.15359/ree.20-1.4

URL: http://www.una.ac.cr/educare

CORREO: educare@una.cr

En el presente artículo presentamos los resultados de la investigación que tuvo como propósito indagar exploratoriamente en torno a los logros educativos alcanzados por personas de origen indígena, afrodescendiente y mestizo o criollo (que popularmente conocemos como "blancos"), y si estos logros se vieron influenciados por este factor identitario o por factores como la localización geográfica, el ingreso temprano al mercado laboral o el tipo de ocupación, que depende, a su vez, de los logros en las otras dimensiones.

Este estudio responde a una inquietud personal como funcionario institucional respecto del estado actual de la educación de la población adulta del país, pues se considera que la educación es un factor que determina muchas dimensiones de la vida de las personas tales como su empleabilidad, movilidad social y los niveles educativos que alcancen las generaciones venideras, y minimiza las tendencias a la exclusión social. Ingresar al sistema educativo y culminar exitosamente los estudios no es una tarea fácil para ninguna persona y se deben sortear muchos obstáculos y barreras, que en algunos casos inducen al abandono de los estudios. Este es resultado de una circularidad estructural que impide alcanzar logros educativos mayores. Tales causas previas son factores que determinan la trayectoria educativa de las personas.

Las explicaciones las debemos buscar en los contextos económicos, sociales y culturales, en los que desarrollan su vida las personas, que determinan las trayectorias vitales y que trascienden la capacidad individual de romper los círculos perversos de la exclusión social y la pobreza (Pérez y Mora, 2007).

El papel tan importante que juega la educación como factor de mejoramiento de la calidad de vida nos lo corrobora el informe del Programa de Naciones Unidas para el Desarrollo (PNUD), que señala que "con una perspectiva de largo plazo, los relativamente bajos niveles de desigualdad (y de pobreza) que presenta actualmente Costa Rica ... se relacionan con los tempranos esfuerzos por brindar educación primaria a toda la población" (Rama,1994, citado en PNUD, 2011, p. 15) "y posteriormente, a partir de la segunda mitad del siglo XX, la expansión de los demás niveles educativos" (Garnier et al., 1991, citado en el PNUD, 2011, p. 15).

En el estudio citado se pone en evidencia mediante la comparación del nivel educativo de los jefes y las jefas de hogar con sus hijos e hijas, que según se alcance primaria, secundaria o universidad, sus descendientes tendrán mejores posibilidades que sus progenitores de alcanzar estudios mayores (PNUD, 2011).

En el mismo informe y mediante el uso de las encuestas de hogares de Costa Rica, se establece que entre los años de 1989 y 2009, se da una ampliación en el logro educativo de las personas entre 18 y 75 años de edad, lo que significa que en el año 2009, para cada rango de edad, la población es más educada y que en 1989, la población entre 18 y 75 años tenía 6,8 grados educativos en promedio, y en el año 2009, este segmento de 18 a 75 tenía 8,6 grados, "para una 'ganancia' educativa de 1,8 grados en los 20 años de diferencia entre encuestas" (PNUD, 2011, p.

2 
16). Todo esto, a pesar de la crisis de los años ochentas que afectó los indicadores en educación, y en especial contrajo la matrícula en secundaria y produjo una mayor diferenciación, social (PNUD, 2013). Esto significa que "una parte importante de la juventud no se matriculó o dejó de asistir a la educación como respuesta a la crisis" (PNUD, 2013, p. 70).

No obstante, los avances alcanzados en la actualidad, aún en el marco de la crisis de los ochentas, entre las personas pobres extremas y no extremas, a partir de la clasificación propuesta por Sauma y Trejos (2014), de entre 15 y 64 años, predominan el empleo en actividades primarias (agricultura y ganadería, entre otras) y secundarias (industria), y entre los vulnerables y no pobres predomina el empleo en el sector de los servicios (comercio), el autoempleo y la actividad por cuenta propia. El predominio de estas ocupaciones se debe básicamente a las deficiencias en cuanto a calificación y a logros educativos menores a secundaria completa (Sauma y Trejos, 2014).

En los hogares de extrema pobreza solo el $8,4 \%$ de personas ocupadas tienen alguna calificación educativa como secundaria, por ejemplo. Es decir, cuanto menos pobres los hogares más calificadas se encuentran las personas: $52 \%$ en los hogares no pobres y ni vulnerables (Sauma y Trejos, 2014).

Entonces, si se puede extender la oferta educativa a mayor número de personas es probable que la pobreza extrema pueda disminuir, esto por cuanto la educación, como dice Sojo (2000), es la base para el acceso a contenidos informativos y simbólicos integradores.

Entonces, ¿constituye un factor de exclusión la identidad étnica y cultural, la localización de estas personas, la inserción temprana al mercado laboral y los tipos de ocupación? Preguntas que este artículo se propone responder.

\section{Antecedentes}

No han sido realizados, a escala nacional, estudios comparativos de los logros educativos alcanzados por indígenas, afrodescendientes y mestizos, sino solo algunas valoraciones generales al respecto, que no desagregan por estas diferencias lo que no hace justicia con la composición culturalmente diversa de la población.

En términos generales, la demanda específica señalada por las organizaciones del mundo indígena nacional se centra en la exigencia de una educación de calidad. Al respecto, la Mesa Nacional Indígena (MNI) ha manifestado que esta no solo no es inclusiva, sino que "los niños, niñas y adolescentes no cuentan con un sistema de enseñanza aprendizaje intercultural. $\mathrm{Ni}$ siquiera cuentan con el recurso mínimo necesario para permanecer (cursivas añadidas) en el sistema educativo" (UNICEF Costa Rica, 2010, p. 45). Induciendo, de este modo, al trabajo infantil y adolescente, ya que las personas jóvenes se ven obligadas a que "cambien las aulas de secundaria por el trabajo, por cierto mal pagado donde además les explotan" (p. 45). 
doi: http://dx.doi.org/10.15359/ree.20-1.4

URL: http://www.una.ac.cr/educare

CORREO: educare@una.cr

En cuanto a los pueblos afrodescendientes, en el estudio del PNUD (López y Delgado, 2013), se concluye que "la dimensión educativa es aquella en que con mayor intensidad se manifiesta el rezago socioeconómico que caracteriza a la población afrodescendiente" (p. 72) y que los resultados educativos del estudio:

Sugieren que, si bien ha habido logros en materia educativa en los últimos años en Costa Rica, todavía persisten múltiples barreras y diversos obstáculos que hay que remover y superar para garantizar la igualdad de oportunidades educativas entre los diversos grupos étnico-raciales del país. (López y Delgado, 2013, p. 72)

Encuanto a la población mestiza, los estudios realizados no se centran en esta característica, sino que por el contrario, estos se generalizan a toda la población sin distinción étnica cultural.

\section{Metodología}

Este estudio descriptivo ha establecido algunos indicadores educativos para estas poblaciones mediante el análisis del X Censo Nacional de Población y VI de Vivienda 2011. En concreto, se establecen las tasas de logro educativo en cuanto a años de estudio y nivel educativo, para distintas poblaciones, según provincia de residencia, tipo de ocupación e inserción temprana al mercado laboral.

Marco conceptual. El logro educativo de una persona, entendido tanto como el número de años de permanencia en el sistema educativo como los niveles educativos que alcance, están determinados por una enorme y diversa cantidad de variables, desde las individuales como el talento o el gusto por el estudio, hasta las de orden social que no dependen de la voluntad de la persona.

En esta dirección, este estudio se ha enfocado en la exploración estadísticas de ciertos factores sociales que se encarnan individualmente, y que pueden operar como obstáculos para acceder a los servicios de educación, como pueden ser la localización geográfica de las personas, según las provincias; y la inserción de menores de edad (10 a 14 y 15 a 19 años) al mercado laboral, mas no la identidad étnico cultural (indígena, afrodescendiente y mestiza).

La presunción básica, al respecto, es que el logro educativo, entendido como el número de años en el sistema educativo y nivel alcanzado, depende de que las variables antes mencionadas no operen como limitantes del acceso a los servicios educativos por parte de las personas independientemente de su identidad étnico-cultutal. Es decir, que la localización geográfica y la inserción temprana al mercado de trabajo no se constituyan en factores de exclusión educativa.

La noción de exclusión educativa está articulada con la noción de exclusión social. Esta hace referencia, tal como lo plantea Gacitúa y Davis (2000), a un conjunto de procesos que contribuyen a aumentar la vulnerabilidad de ciertos grupos sociales frente a factores de riesgo

4

Marcelo Gaete-Astica

(c) (1) (3) 
que pueden llevarlos a una situación de pobreza y vulnerabilidad social. Gacitúa y Davis (2000) definen la exclusión social "como la imposibilidad de un sujeto o grupo social para participar efectivamente a nivel económico, social, cultural, político e institucional" (p. 14).

Por otra parte, tal como lo señalan Pérez y Mora (2007), la problemática de la exclusión social hace referencia al proceso que produce desigualdades sociales, "la exclusión es la manifestación más extrema de la desigualdad social" (p. 30). "Es decir, la exclusión nunca es absoluta, sino más bien relativa. Y, por tanto, los excluidos se encuentran solo donde pueden estar, en la propia sociedad, aunque estén a su manera" (Karsz, 2004 en Pérez y Mora, 2007, p. 31). Por lo demás, la exclusión es un fenómenos dinámico, que muestra que las personas tanto de forma individual como colectiva se mueven para salir de tal condición, la de exclusión (Pérez y Mora, 2007). La educación es una de esos medios para salir de esta situación.

El logro educativo (años de estudios y niveles educativos alcanzados) depende no solo de la disposición individual sino de la articulación de diversas variables sociales que pueden facilitarlo u obstaculizarlo. A su vez, la educación es precisamente un factor de cambio y movilidad social importante, que define en mucho los logros que en ese terreno alcancen las personas.

La ausencia de educación y de calificación es uno de los factores de exclusión social, pues limita el acceso a oportunidades de empleo mejor remuneradas (Pérez y Mora, 2007). Este tipo de desventaja educativa, por su parte, excluye también a personas que son parte de colectivos sociales que han sufrido, previamente, de exclusión como, por ejemplo, indígenas, migrantes, afrodescendientes y residentes de zonas rurales.

En este sentido, siguiendo a Perry (2000), se ha observado también que la pobreza y la exclusión social están ligadas con la etnia, la organización social y la cultura, en articulación con la distribución de los ingresos:

Los grupos o sectores sociales excluidos pueden ser múltiples, pero son dos grupos amplios los que se observan de una forma más intensa. Uno es el grupo de los indígenas, y el otro, en algunos países, es el conformado por gente con ascendencia africana -los afrolatinos. Esto no quiere decir que no haya otras líneas de exclusión, tales como sexo, edad o religión en algunos países. Lo que sí parece querer decir, es que las diferencias étnicas y raciales se encuentran entre los factores más evidentes de la exclusión social. (p. 10)

Perry (2000) enfatiza más adelante que:

El reducido acceso de los grupos excluidos a la escolaridad y los servicios de salud explica en parte la baja capacidad futura de aprendizaje, los menores salarios y la limitada participación política. El bajo acceso a la escolaridad, a su vez, también puede ser explicado por la baja participación política, la pobreza de los padres y una total discriminación en el mercado de trabajo. (p. 11) 
doi: http://dx.doi.org/10.15359/ree.20-1.4

URL: http://www.una.ac.cr/educare

CORREO: educare@una.cr

Estos procesos excluyentes en lo social y educativo condicionan, en una medida muy alta, la capacidad familiar de salir adelante y superar los umbrales de pobreza, toda vez que la pérdida sistemática de capacidad de integración entre generaciones van generando, cada vez, más potencial de exclusión y menos capacidad de educabilidad familiar.

Es decir, la reproducción familiar en términos sociales y económicos depende también de la capacidad que tengan las familias de reproducir de modo ampliado su educabilidad (es decir, su potencial de ser educado o educada), lo que tiene que ver tanto con la capacidad propia de la familia, como la calidad de la oferta de educación de la educación pública y los medios de compensación de que disponga esta. Así, siguiendo a Bourdieu (1997):

La reproducción de la estructura de la distribución del capital cultural se opera en la relación entre las estrategias de las familias y la lógica específica de la institución escolar. Ésta tiende a proporcionar el capital escolar, que otorga bajo la forma de títulos (credenciales), al capital cultural detentado por la familia y transmitido por una educación difusa o explicita en el curso de la primera educación. (p. 108)

Y esto lo hemos visto aquí, cuando las familias deciden retirar a sus hijos de la educación, porque el costo de esta, incluso en el sector de educación pública gratuita, es más alto que lo que podrían derivar a futuro terminando sus estudios. Disyuntiva que se opera más rápidamente cuando el joven o la joven no cree que vaya a pasar el año.

Al revés de lo que sucede en el estudio sobre Japón, señala Bourdieu (1997), que el cálculo racional de las familias respecto de la educación pasa por las expectativas de movilidad social ascendente. Por el contrario, en nuestro país, las familias deciden no gastar más en educación, si mediante el trabajo adolescente se pueden generar más ingresos.

Claro, la educación en cuanto calidad y cantidad se agrega como plus valor al capital cultural que tiene la familia como tal, si esta es económicamente pobre y recibe un pobre capital escolar.

Entonces, tal como cualquier fenómeno sistémico, la familia entra en crisis de valor sociocultural, que se traduce, claro está, en disvalor económico para la familia. Y, como en el contexto de mercado el valor se traduce a dinero, la imposibilidad de las familia de comprar más capital escolar les impide, globalmente, aumentar su capital cultural; por ende, una situación integral de pobreza (social, económica y cultural) se entroniza de tal modo en esa estructura que las ayudas son insuficientes, tampoco sirven, en este sentido, los programas compensatorios como Avancemos del Instituto Mixto de Ayuda Social (IMAS) o el del Fondo Nacional de Becas (FONABE), para el caso local. Tales recursos solo ayudan a comprar la educación que se pueda 
pagar, so pena de que el mercado de trabajo ofrezca costos de oportunidad mejores para la mayoría de miembros de la familia, incluido las personas menores de edad de las familias en pobres y extrema pobreza.

En resumen, hay factores de exclusión social y laboral que limitan el acceso a la educación, y un logro educativo bajo es, a su vez, factor de exclusión. Del mismo modo, vivir en regiones deprimidas y que se localizan fuera de las inversiones productivas se constituye en un factor de exclusión y, en este predicamento, están grupos indígenas y afrodescendientes.

En resumen, la exploración de esta temática requiere de aproximaciones sucesivas y graduales que permitan elaborar explicaciones correlacionales determinantes para poder intervenir por medio de políticas públicas pertinentes.

En este estudio, se ha decidido conocer parcialmente las características que adoptan tres de las tantas variables que juegan un rol importante, cuales son la identidad étnica cultural, la localización provincial de la población en estudio y la inserción temprana en el mercado laboral.

\section{Discusión de resultados}

Este estudio se ha planteado el objetivo de conocer las tasas de logro de distintos colectivos étnico culturales para determinar, si algunos factores relevante son causales de las diferencias, si estas se presentan.

La comparación que se ha realizado ha buscado diferenciar los logros educativo de indígenas, afrodescendientes y mestizos, esta última categoría en lenguaje normal se identifica con los "blancos", concepto que hoy ha sido desechado por constituir una abstracción racista que no da cuenta de la realidad social y cultural de la sociedad costarricense actual, en la que los procesos de mestizaje han sido procesos regulares en la historia.

Estudiar es un bien preciado socialmente por cuanto ya ha sido señalado, en este estudio, así como en muchos otros, que este es un recurso que mejora las condición de inserción laboral y por esa vía se contrarresta las tendencia a la exclusión social, y les permite a las personas pertenecientes a las minoría indígenas y afrodescendientes trazar una ruta de progreso personal y colectivo.

Al respecto, un supuesto de este estudio, la hipótesis de investigación, es la de que la identidad étnica es un factor de exclusión educativo, y que junto con el factor de localización residencial y el ingreso temprano al mundo del trabajo se pueden observar diferencias sustanciales entre una población y otra, siendo las más beneficiada en este contexto la mestiza. Sin embargo, una primera conclusión, a la que hemos llegado, es que el acceso a los servicios educativos no está determinado por la diferencia cultural entre las población, como se puede observar en las figuras 4, 5 y 6 en donde los porcentajes parecidos de personas pertenecientes 
doi: http://dx.doi.org/10.15359/ree.20-1.4

URL: http://www.una.ac.cr/educare

CORREO: educare@una.cr

a estos grupos alcanzan sexto grado, lo que nos informa de que no hay discriminación por identidad étnica y cultural.

Ello, por cuanto se pudo establecer que la gran mayoría de personas con independencia de su condición étnica y cultural logra estar al menos 6 años en el sistema educativo, y logran obtener el sexto grado, y son muchas menos las personas que alcanzan noveno, undécimo o duodécimo, y todavía menos las que logran estar en la universidad, las diferencias entre poblaciones son mínimas en estos logros.

Son, más bien, los factores de localización y la inserción temprana al mundo del trabajo los que parecen tener un peso mayor como diferenciadores, así las pocas diferencias que se observan a favor de mestizos, seguidos por afrodescendientes y luego indígenas se dan principalmente por factores de residencia, siendo lo que viven en el Valle Central los más afortunados si quieren alcanzar más de sexto grado, y tener al menos undécimo y duodécimo, pero se da la circunstancia de que estos niveles educativos están restringidos para la mayoría de las personas, pero particularmente, para las indígenas.

\section{Logro educativo de la población afrodescendiente, indígena y mestiza, y factores de localización e inserción temprana al mundo del trabajo}

El logro educativo entendido como una medida que combina la cantidad total de años de permanencia en el sistema educativo y el nivel educacional alcanzado, se calculó para las tres poblaciones que se han estudiado en esta investigación. A continuación se revisan, entonces, los logros alcanzados por grupos indígenas, afrodescendientes y mestizos, según la localización de estas poblaciones, la inserción temprana al mundo del trabajo y, finalmente, se realizará un alcance a los tipos de ocupación.

\section{Logro educativo e identidad sociocultural}

El número de años de educación de personas de indígenas $-6,1-$, afrodescendientes -7años-, y mestizos -7,8-, muestra variaciones mínimas entre ellas. Aunque las indígenas alcanzan en promedio ${ }^{2}$ un año menos, que afrodescendientes y mestizas. La moda es, sin embargo, de 6 años para todas las poblaciones, lo que probablemente indica que la gran mayoría de personas tiene los 6 grados de enseñanza primaria. Luego, el número de años que se alcanza, va decreciendo en todos los casos. Son muy pocas personas las que logran más de 6 años, como se puede ver en la figura 1:

2 Estimaciones hechas por medio del promedio ponderado, primero multiplicando el número de años por la frecuencia especifica y sumando todos estos valores; y luego dividiendo este total entre la sumatoria total de las frecuencias de los años de estudio.

8 


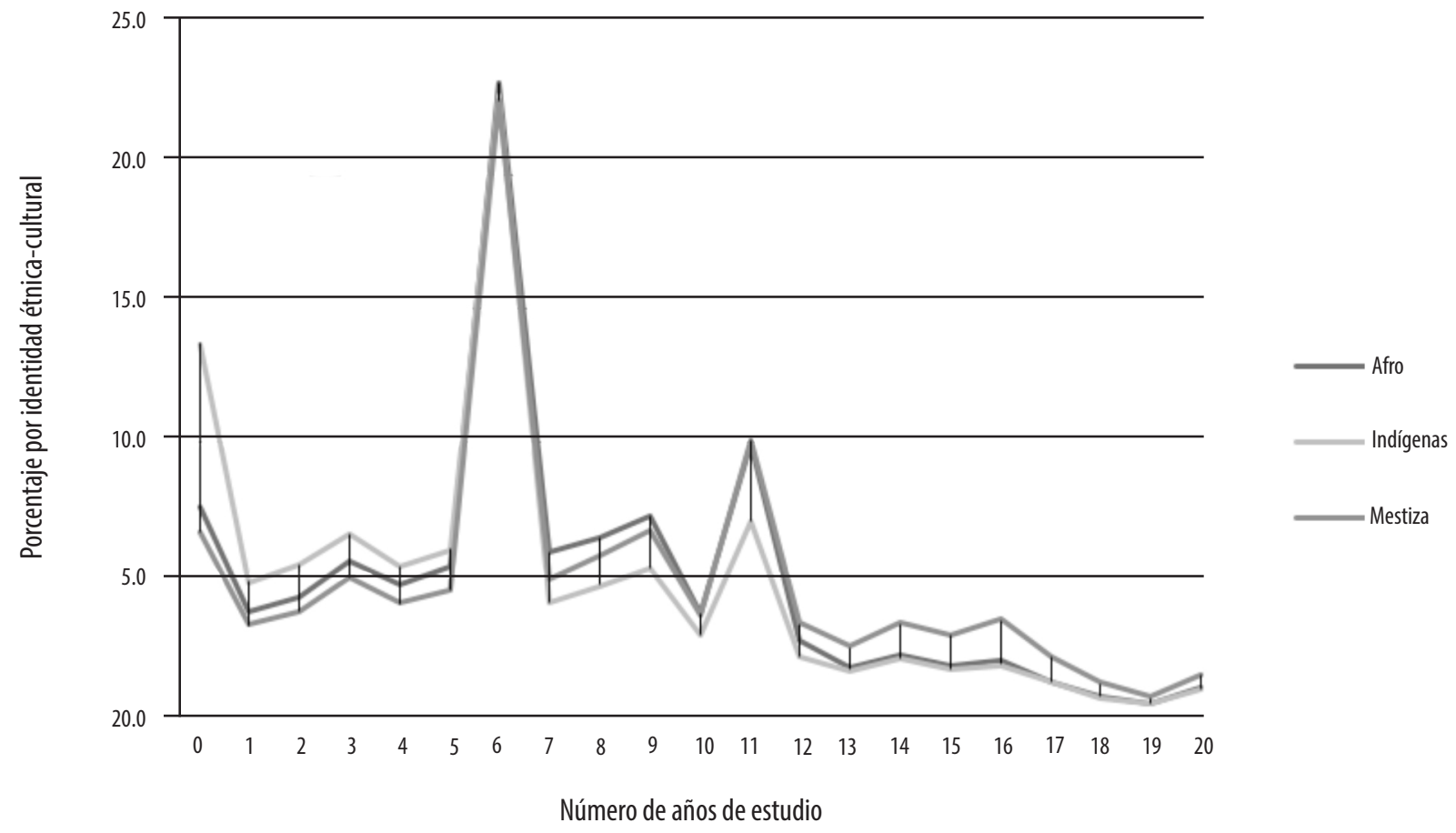

Figura 1. Número de años de estudio por identidad étnico-cultural. Elaboración propia con base en el Censo (INEC, 2011).

En cuanto al nivel educativo alcanzado por estas poblaciones, es VI grado el nivel educativo alcanzado por 23 de cada cien personas entre 5 y 85 años de edad, de los colectivos étnicos y culturales estudiados. Variaciones un poco más relevantes se observan en las personas que terminaron secundaria académica, tenemos, en este nivel, que del total de personas afrodescendientes, mestizas e indígenas, el mayor número de personas que terminaron este nivel corresponde a la categoría de afrodescendientes con 12,3\%, le sigue la de mestiza con 9,7\% para culminar con la población indígena con 7,0\%.

En la figura 2 se muestra comparativamente esta situación.

La figura 2 muestra que mientras la educación primaria está generalizada, aunque la matricula de la población indígena es, relativamente, mayor; en secundaria, el logro mayor lo alcanza la población afrodescendiente. $Y$ en los grados posteriores a secundaria aumenta el logro de la población mestiza. No obstante, las diferencias entre todos estos grupos no indican que el factor étnico-cultural sea una variable que determina ostensiblemente las diferencias en cuanto a acceso a educación, más que la condición de exclusión social y la de pobreza. 
doi: http://dx.doi.org/10.15359/ree.20-1.4

URL: http://www.una.ac.cr/educare

CORREO: educare@una.cr

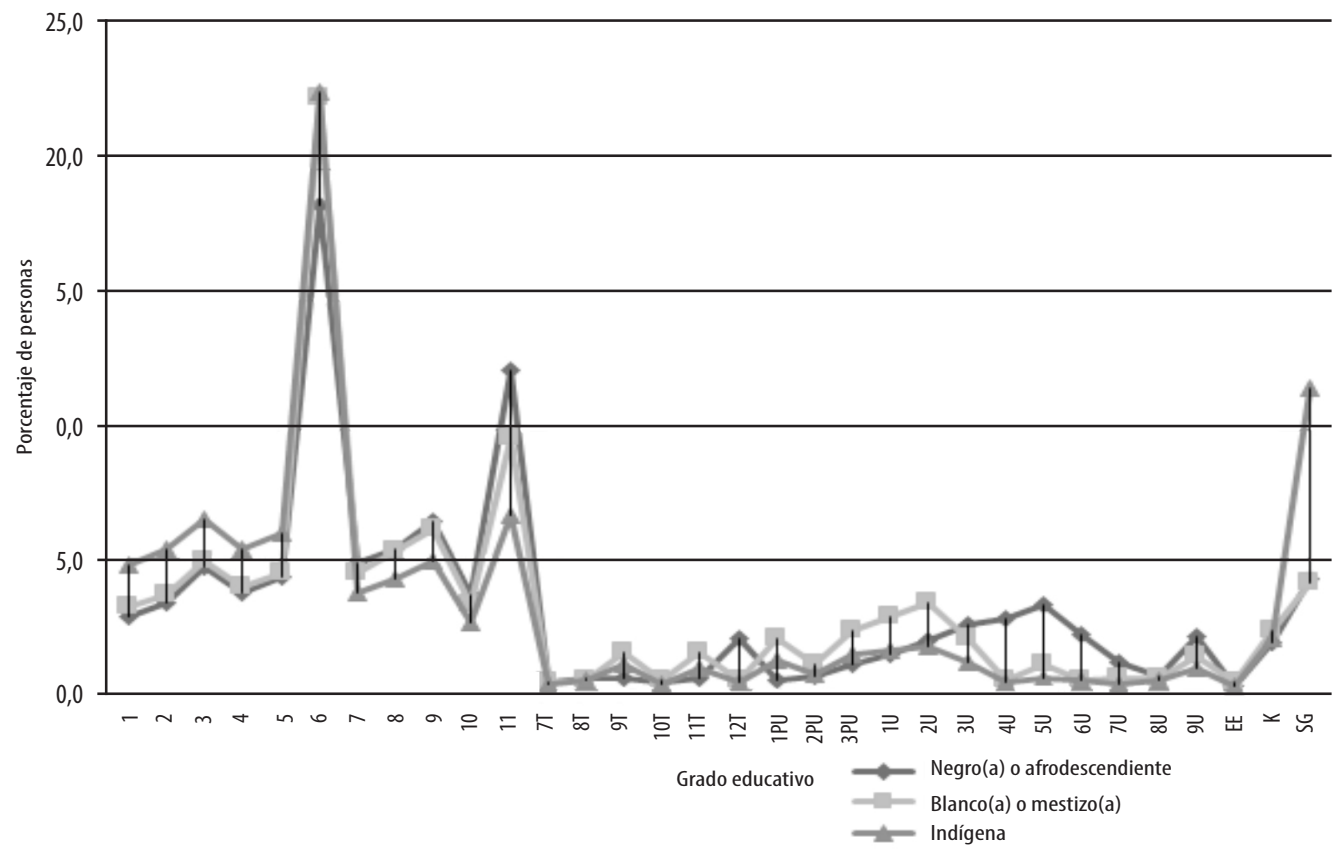

Figura 2. Grado educativo alcanzado por la población afrodescendiente, indígena y mestiza, según el censo del año 2011. Elaboración propia con base en el (INEC, 2011).

\section{Logro educativo según variables de localización}

Una mirada panorámica de estos datos, como lo enseña la tabla 1, enfoca el hecho de que en todas las provincias la mayoría de estas personas, de las distintas poblaciones étnicas culturales, no parece haber cursado ningún año en educación. Le siguen las que han cursado 1 y 6 años, siendo más relevante el sexto. De este punto de corte ${ }^{3}$ hacia los restante se ve, como era de esperarse, una disminución en la cantidad de años cursados. Entonces, que la mayoría haya estado 6 años en educación es indicativo de que la enseñanza primaria, que es una oferta con cobertura casi de $100 \%$, es lo que un número importante de las personas ha alcanzado.

${ }^{3}$ La presentación de los años de estudios alcanzados por estas poblaciones, residentes en las siete provincias, se ha realizado mediante el establecimiento de puntos de corte específicos $-0,1,6,12,18$ y 19 años-, que corresponden al primer y último año de primaria, el ingreso y terminación de secundaria y los años universitarios. Estos punto de corte no significan que las personas terminaron esos niveles (para eso se tiene los datos de nivel educativo), sino que indican la cantidad de años en el sistema educativo. Se hizo de esta manera, simplemente para resumir la información. 
doi: http://dx.doi.org/10.15359/ree.20-1.4

URL: http://www.una.ac.cr/educare

CORREO: educare@una.cr

En la tabla 1 se presentan porcentualmente las variaciones en cuanto a años de estudio por provincias.

Tabla 1

Población de personas indígenas, afrodescendientes y mestizas por años de estudios cursados y provincias (Datos relativos)

\begin{tabular}{|c|c|c|c|c|c|c|c|c|c|c|c|c|c|c|c|c|c|c|c|c|c|}
\hline & & 0 años & & & 1 & & & 6 & & & 12 & & & 18 & & & 19 & & & 20 & \\
\hline 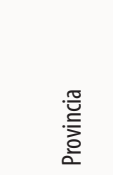 & 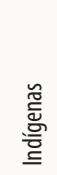 & 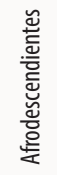 & $\begin{array}{l}\stackrel{\widetilde{N}}{\bar{E}} \\
\stackrel{\tilde{E}}{\Sigma}\end{array}$ & 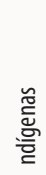 & 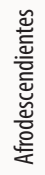 & $\begin{array}{l}\stackrel{\mathscr{N}}{\tilde{E}} \\
\stackrel{\tilde{\omega}}{\Sigma}\end{array}$ & 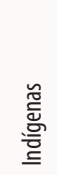 & 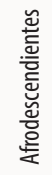 & $\begin{array}{l}\stackrel{\tilde{N}}{\bar{N}} \\
\stackrel{\tilde{\omega}}{\Sigma}\end{array}$ & 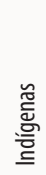 & 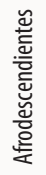 & 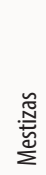 & 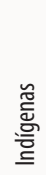 & 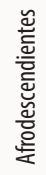 & $\begin{array}{l}\stackrel{\tilde{N}}{\tilde{E}} \\
\stackrel{\tilde{E}}{\Sigma}\end{array}$ & 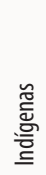 & 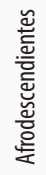 & $\begin{array}{l}\stackrel{\widetilde{N}}{\bar{E}} \\
\stackrel{\tilde{E}}{\Sigma}\end{array}$ & 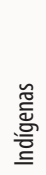 & 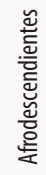 & 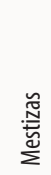 \\
\hline Alajuela & 9,9 & 8,1 & 24,1 & 3,8 & 3,4 & 10,9 & 24,3 & 21,9 & 9,4 & 2,3 & 2,5 & 1,0 & 0,6 & 0,9 & 15,6 & 0,6 & 0,6 & 18,4 & 0,8 & 1,5 & 20,5 \\
\hline Cartago & 18,8 & 7,6 & 21,0 & 4,8 & 3,9 & 10,0 & 21,9 & 21,4 & 11,3 & 1,7 & 2,5 & 1,5 & 0,5 & 1,0 & 15,5 & 0,5 & 0,4 & 19,0 & 0,6 & 1,2 & 21,6 \\
\hline Guanacaste & 6,5 & 7,4 & 23,1 & 3,5 & 5,0 & 11,3 & 24,6 & 20,1 & 8,3 & 3,3 & 2,7 & 1,0 & 0,6 & 1,4 & 15,9 & 0,6 & 0,1 & 19,6 & 1,1 & 2,1 & 20,9 \\
\hline Heredia & 5,8 & 9,8 & 18,6 & 2,4 & 3,6 & 8,7 & 21,1 & 23,4 & 15,3 & 3,2 & 1,9 & 2,4 & 1,6 & 1,0 & 14,8 & 1,6 & 0,6 & 18,0 & 1,5 & 1,1 & 22,3 \\
\hline Limón & 20,8 & 11,0 & 27,1 & 5,6 & 2,5 & 13,6 & 22,2 & 30,5 & 4,2 & 1,3 & 0,8 & 0,6 & 0,1 & 1,7 & 16,8 & 0,1 & 0,8 & 18,4 & 0,3 & 0,0 & 19,2 \\
\hline Puntarenas & 16,2 & 9,5 & 26,5 & 7,0 & 2,3 & 12,4 & 22,3 & 25,4 & 5,5 & 1,3 & 2,0 & 0,8 & 0,2 & 1,0 & 16,3 & 0,2 & 0,1 & 18,9 & 0,3 & 1,6 & 19,5 \\
\hline San José & 8,1 & 5,5 & 17,5 & 2,7 & 2,5 & 8,6 & 22,9 & 16,8 & 13,5 & 2,7 & 4,2 & 2,1 & 0,9 & 1,2 & 15,4 & 0,9 & 0,6 & 18,8 & 1,8 & 2,4 & 24,1 \\
\hline
\end{tabular}

Nota: Elaboración propia con base en el Censo Nacional de Población (INEC, 2011).

Limón ${ }^{4}$ es la provincia en la que más indígenas no presentan ningún año de estudio, seguido de Cartago y Puntarenas. No obstante, que este cálculo se ha realizado con el total de personas indígenas, incluidas personas menores de cinco años, el número siempre resulta alto en comparación con las otras poblaciones. Por otro lado, una cuarta parte de estas personas tiene seis años de estudio.

\footnotetext{
${ }^{4}$ Los porcentajes se han calculado con base en la población indígena total reportada para cada provincia por el Censo INEC (2011): Alajuela, 11383; Cartago, 11484; Guanacaste, 14482; Heredia, 6625; Limón, 36034; Puntarenas, 33537 y San José, 29351. En el caso de las personas afrodescendientes, los datos son: Alajuela, 8055; Cartago, 2485; Guanacaste, 1445; Heredia, 1994; Limón, 118; Puntarenas, 696; San José, 48410. Y en el caso de las personas mestizas tenemos: Alajuela, 316.285; Cartago, 176.460; Guanacaste, 109.952; Heredia, 156.160; Limón, 125954; Puntarenas, 140.841; San José, 508.748.
} 
doi: http://dx.doi.org/10.15359/ree.20-1.4

URL: http://www.una.ac.cr/educare

CORREO: educare@una.cr

En las provincias de Alajuela, San José y Guanacaste las personas indígenas han cumplido con al menos 6 años de estudio. En cuanto a haber alcanzado 12 años sobresalen, por un escaso punto porcentual, Heredia y Guanacaste. Pareciera que para tener más años de estudio habría que salir de Limón y, probablemente, en particular de Talamanca.

En cuanto a la población de personas afrodescendientes, tenemos que la norma también es haber cursado 6 años. Mientras que muchas menos personas alcanzan 12, 18, 19 o 20 años de estudio.

Limón encabeza la estadística de mayor número de personas con 0 y 6 años de educación, seguido por Puntarenas, y luego el resto de las provincias. Es San José la que ocupa el último lugar.

Pero, por otro lado, San José presenta el mayor número de personas con 12 y 20 años de estudios. Parece que la población afrodescendiente, si quiere alcanzar sexto grado, debiera vivir principalmente en Limón, y no en San José. Y para tener más de 6 años en el sistema educativo, debiera vivir en San José.

La población de personas mestizas, comparativamente con las otras poblaciones, también alcanza 6 años de educación. Queda relativamente de manifiesto, por otro lado, que alcanzar más años de estudio es más probable si la persona es mestiza.

Heredia y San José son las provincias que tienen más personas con 6 y 12 años de estudios, respectivamente. De forma interesante, hay un número relativamente alto de personas con 18 , 19 y 20 años de estudios, en todas las provincias.

En cuanto al nivel educativo alcanzado, medido como la finalización de algún nivel educativo, la información revisada indica que el sexto grado es el que más personas alcanzan, alrededor del $21 \%$-indistintamente de la identidad sociocultural de pertenencia, y la provincia donde residen-.

Luego de sexto, que es un logro que alcanza alrededor de un $21 \%$ de la población, el porcentaje de personas que aprueban noveno y décimo decae notablemente. En promedio, estos porcentajes son 5 y $7 \%$ en el caso de indígenas, $6 \%$ y $10 \%$ en el de las personas afrodescendientes y $6 \%$ y $8 \%$ para las personas mestizas.

Es decir, a la población indígena se le dificulta alcanzar niveles educativos superiores en Limón y Puntarenas. En el caso de las personas afrodescendientes, encontramos que para el nivel de noveno respecto de sexto baja el número de personas con ese grado, y 
se mantiene estable el porcentaje de personas que alcanzan undécimo. La posibilidad de tener al menos 5 años de enseñanza universitaria disminuye en todas las provincias; pero se observa que hay más probabilidades de alcanzar estos 5 años de universidad si se vive en Cartago, Heredia y San José que en las otras provincias; esto probablemente obedece a la mayor oferta de estudios universitarios.

En relación con el acceso a la educación secundaria, el logro en noveno ronda una tasa de $6 \%$, situándose en un valor intermedio entre el logro entre la población indígena y la afrodescendiente. En cuanto a educación universitaria, alrededor del 3\% de esta población alcanzan al menos 5 años de universidad.

Desde la perspectiva geográfica, con las provincias como el vector del análisis, no se evidencian diferencias significativas de logro educativo entre las poblaciones, sino más bien ciertas ventajas que tiene para una población alcanzar cierto logro, si vive en una provincia u otra. Y también cierta desventaja.

\section{Inserción temprana al mercado laboral y poblaciones étnicas culturales}

La exploración en torno a la inserción en el mercado de trabajo de las poblaciones que hemos venido estudiando se hará por medio de una estimación general del nivel de ocupación de estas poblaciones, para luego examinar la ocupación entre las personas de entre 10 y 14 años, y las de 15 y 19. La idea es mostrar cómo hay personas que debieran estudiar y no trabajar, comparando entre personas indígenas, afrodescendientes y mestizos.

En la tabla 2 podemos observar, comparativamente, que en las tres poblaciones el nivel de ocupación se ubicó en más de un $65 \%$, entre la población masculina de los grupos indagados (indígenas 60,7\%, afrodescendientes 64,1\% y mestizos 65,2\%).

En cuanto a la población femenina de estas poblaciones, se observan porcentajes de ocupación muchísimos más bajos que los de los hombres: 24,8\% entre las indígenas, 37,4\% entre las personas afrodescendientes y $33,1 \%$ entre las mestizas. Menos mujeres indígenas están ocupadas que en el caso de las otras mujeres. Lo que podría deberse, probablemente, a las pocas oportunidades de empleo en los territorios indígenas. La desocupación afectó, en la fecha del censo, a $2 \%$ de la población masculina de indígenas, afrodescendientes y mestizos. Veamos la tabla 2: 
doi: http://dx.doi.org/10.15359/ree.20-1.4

URL: http://www.una.ac.cr/educare

CORREO: educare@una.cr

Tabla 2

Condición de actividad de la población indígena, afrodescendiente y mestiza, en el momento del X Censo Nacional de Población y Vivienda. Censo Nacional

\begin{tabular}{|c|c|c|c|c|c|c|c|c|c|}
\hline & $\begin{array}{l}\text { 음 } \\
\text { 을 }\end{array}$ & 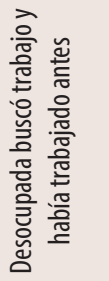 & 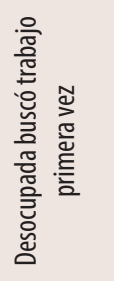 & 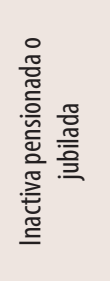 & 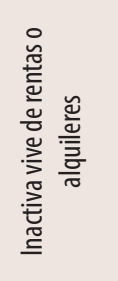 & 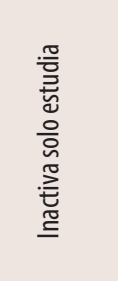 & 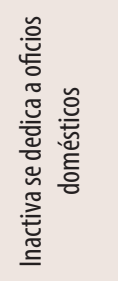 & 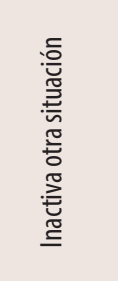 & 吾 \\
\hline \multicolumn{10}{|c|}{ Indígena } \\
\hline Hombre & 25.262 & 836 & 114 & 2.983 & 124 & 6.050 & 1.198 & 5.037 & 41.604 \\
\hline Mujer & 10.174 & 295 & 67 & 1.952 & 67 & 5.813 & 18.886 & 3.834 & 41.088 \\
\hline \multicolumn{10}{|c|}{ Afrodescendiente } \\
\hline Hombre & 12.989 & 533 & 66 & 1.433 & 78 & 2.779 & 287 & 2.111 & 20.276 \\
\hline Mujer & 6.861 & 262 & 54 & 1.079 & 47 & 2.713 & 5.409 & 1.932 & 18.357 \\
\hline \multicolumn{10}{|c|}{ Mestiza } \\
\hline Hombre & 910.989 & 28.376 & 3.886 & 91.105 & 4.012 & 235.316 & 14.328 & 110.139 & 1.398 .151 \\
\hline \multirow[t]{2}{*}{ Mujer } & 499.274 & 12.501 & 2.699 & 67.672 & 3.546 & 260.038 & 558.174 & 106.074 & 1.509 .978 \\
\hline & $\%$ & $\%$ & $\%$ & $\%$ & $\%$ & $\%$ & $\%$ & $\%$ & $\%$ \\
\hline \multicolumn{10}{|c|}{ Indígena } \\
\hline Hombre & 60,7 & 2,0 & 0,3 & 7,2 & 0,3 & 14,5 & 2,9 & 12,1 & 100,0 \\
\hline Mujer & 24,8 & 0,7 & 0,2 & 4,8 & 0,2 & 14,1 & 46,0 & 9,3 & 100,0 \\
\hline \multicolumn{10}{|c|}{ Afrodescendiente } \\
\hline Hombre & 64,1 & 2,6 & 0,3 & 7,1 & 0,4 & 13,7 & 1,4 & 10,4 & 100,0 \\
\hline Mujer & 37,4 & 1,4 & 0,3 & 5,9 & 0,3 & 14,8 & 29,5 & 10,5 & 100,0 \\
\hline \multicolumn{10}{|c|}{ Mestiza } \\
\hline Hombre & 65,2 & 2,0 & 0,3 & 6,5 & 0,3 & 16,8 & 1,0 & 7,9 & 100,0 \\
\hline Mujer & 33,1 & 0,8 & 0,2 & 4,5 & 0,2 & 17,2 & 37,0 & 7,0 & 100,0 \\
\hline
\end{tabular}

Nota: Elaboración propia con base en datos del Censo Nacional de Población y Vivienda (INEC, 2011). 
En resumen, al menos para esta fecha no se observan diferencias sustantivas entre las personas indígenas, afrodescendientes y mestizas en su condición de ocupación según los rasgos identitarios. Alrededor del $60 \%$ de hombres y más del $25 \%$ de las mujeres se encontraban ocupadas. Las mujeres mestizas y afrodescendientes presentan mayores tasas de ocupación.

En cuanto a la inserción en el mercado laboral de personas menores de edad, de entre 10 y 19 años, distribuidas en dos segmentos 10 a 14 y 15 a 19 años, estaban, en el año 2011, ocupadas y no estudiando, 3.643 y $63.251,-0,9 \%$ y $15,6 \%$ respectivamente- en relación con el total de estas personas de esas edades. Se trata de personas excluidas del sistema educativo y que debieron ingresar al mundo del trabajo.

En condición a la vez de ocupadas en el mercado laboral y excluidas de educación se encontraban 197 personas indígenas de entre 10 y 14 años y 1.467 de entre 15 y 19 años personas (3,43\% y $16,13 \%$ respectivamente); 37 y 537 personas afrodescendientes $(1,8 \%$ y $14,2 \%)$ y 2.865 y 52.347 personas mestizas $(1,5 \% \text { y } 15,5 \%)^{5}$. Ambos rangos de edad se refieren a personas menores de edad, que según la legislación nacional debieran estar estudiando (ver tabla 3).

En resumidas cuentas, es en las personas indígenas en donde, proporcionalmente, podemos encontrar más personas insertas en el mundo del trabajo, en edades en que se supone debieran estar estudiando, les siguen las personas afrodescendientes y luego las mestizas.

Estos datos permiten, entonces, señalar que ser indígena y probablemente vivir en el territorio ancestral es un factor que dificulta continuar los estudios, y se facilita el ingreso al mercado de trabajo. En una medida parecida, esto también le sucede a las personas afrodescendientes, sobre todo en secundaria. Las personas mestizas, por su parte, tienen más opciones de estudiar y es por ello que, proporcionalmente, a partir de los datos presentados, las personas ocupadas son relativamente menos.

Una aproximación a esta realidad del abandono del estudio, nos la brinda la Encuesta Nacional de Hogares (ENAHO, INEC, 2011), que indaga las razones de no asistencia a educación de jóvenes entre 10 y 17 años.

\footnotetext{
${ }_{5}^{5}$ El total de población indígena, afrodescendiente y mestiza, por los rangos de edad, es: Indígenas de 10 a 14 y 15 a 19, 5.741 y 9.095 respectivamente; afrodescendientes 2.091 y 3.770; mestizas 193.049 y 338.061 .
} 
doi: http://dx.doi.org/10.15359/ree.20-1.4

URL: http://www.una.ac.cr/educare

CORREO: educare@una.cr

\begin{tabular}{|c|c|c|c|c|c|c|c|c|c|c|}
\hline & 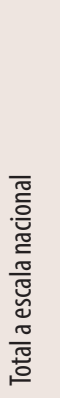 & 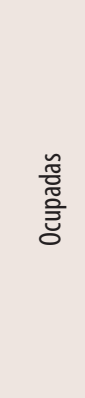 & 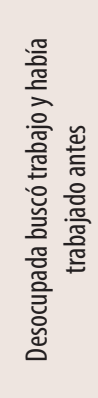 & 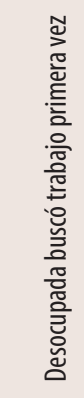 & 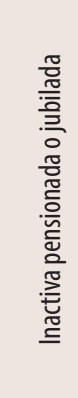 & 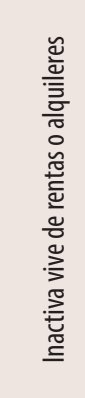 & 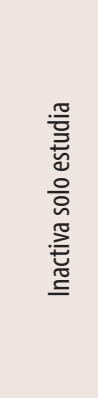 & 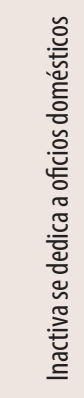 & 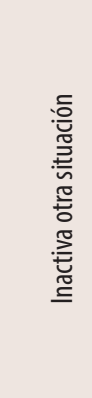 & 要 \\
\hline De 10 a 14 años & & 3.643 & 138 & 113 & 81 & 109 & 205.599 & 5.981 & 17.108 & 232.772 \\
\hline De 15 a 19 años & & 63.251 & 4.855 & 2.984 & 242 & 169 & 256.773 & 35.844 & 41.058 & 405.176 \\
\hline
\end{tabular}

Total por identidad étnica y cultural

\begin{tabular}{l|c|c|c|c|c|c|c|c|c|}
\multicolumn{1}{c}{ Indígenas } \\
\hline De 10 a 14 años & 197 & 4 & 10 & 1 & 2 & 4.646 & 374 & 507 & 5.741 \\
De 15 a 19 años & 1.467 & 81 & 61 & 4 & 3 & 4.784 & 1.587 & 1.108 & 9.095 \\
\hline
\end{tabular}

Afrodescendientes

\begin{tabular}{l|r|c|c|c|c|c|c|c|c|c|c}
\hline De 10 a 14 años & 37 & 1 & 2 & 2 & 1 & 1.794 & 46 & 208 & 2.091 \\
\hline De 15 a 19 años & 537 & 39 & 37 & 0 & 2 & 2.338 & 282 & 535 & 3.770 \\
\hline
\end{tabular}

Total relativo por identidad étnica y cultural

\begin{tabular}{|c|c|c|c|c|c|c|c|c|c|}
\hline \multicolumn{10}{|c|}{ Indígenas } \\
\hline De 10 a 14 años & 3,4 & 0,1 & 0,2 & 0,0 & 0,0 & 80,9 & 6,5 & 8,8 & 100 \\
\hline De 15 a 19 años & 16,1 & 0,9 & 0,7 & 0,0 & 0,0 & 52,6 & 17,4 & 12,2 & 100 \\
\hline \multicolumn{10}{|c|}{ Afrodescendientes } \\
\hline De 10 a 14 años & 1,8 & 0,0 & 0,1 & 0,1 & 0,0 & 85,8 & 2,2 & 9,9 & 100 \\
\hline De 15 a 19 años & 14,2 & 1,0 & 1,0 & 0,0 & 0,1 & 62,0 & 7,5 & 14,2 & 100 \\
\hline \multicolumn{10}{|c|}{ Mestizas } \\
\hline De 10 a 14 años & 1,5 & 0,1 & 0,0 & 0,0 & 0,0 & 88,9 & 2,4 & 7,0 & 100 \\
\hline De 15 a 19 años & 15,5 & 1,2 & 0,7 & 0,1 & 0,0 & 64,3 & 8,5 & 9,7 & 100 \\
\hline
\end{tabular}

Nota: Elaboración propia con base en el Censo Nacional (INEC, 2011). 
doi: http://dx.doi.org/10.15359/ree.20-1.4

URL: http://www.una.ac.cr/educare

CORREO: educare@una.cr

En la figura 3, se observa que el porcentaje más alto de no asistencia es la falta de interés en el estudio, lo cual alerta de la posible falta de pertinencia de la oferta educativa para esta población de jóvenes.

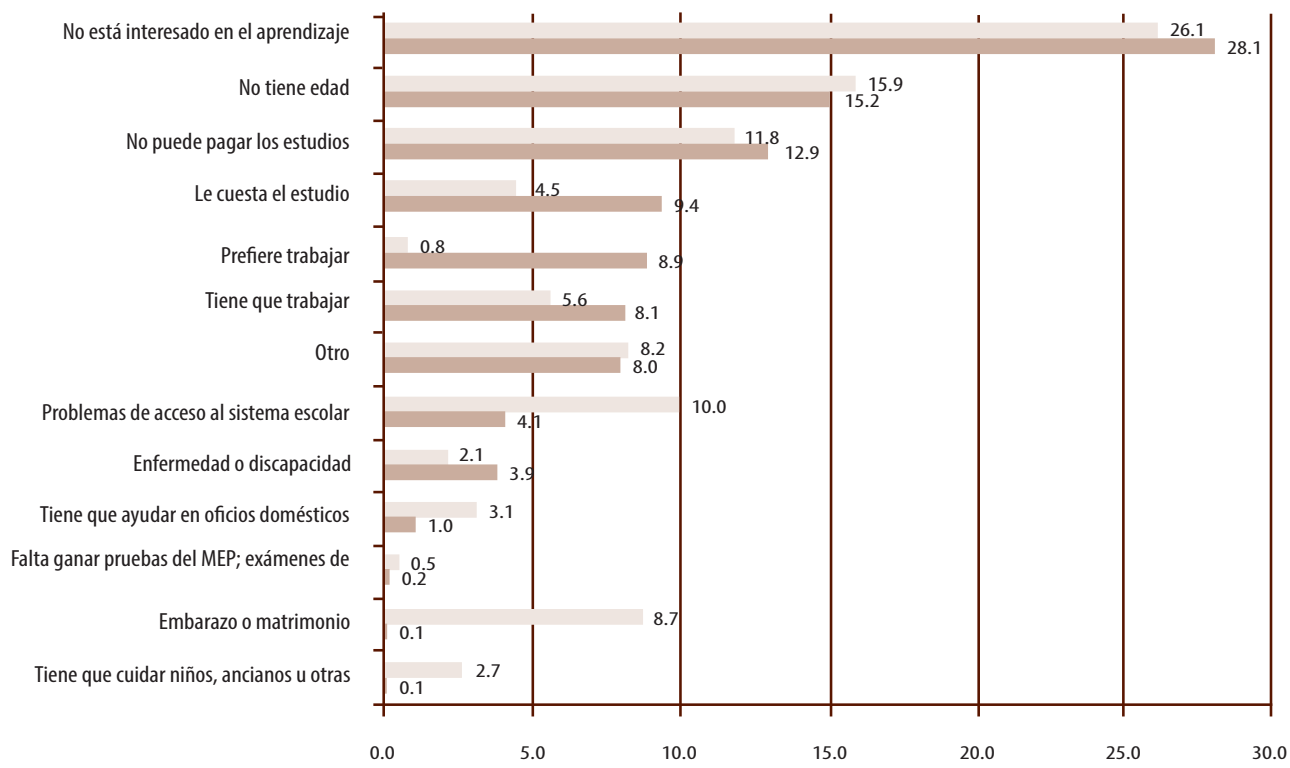

Figura 3. Razones para no estudiar brindadas por personas de ambos sexos de edades entre 10 y 17 años en el año 2011. Elaboración propia con base en la Encuesta Nacional de Hogares (ENAHO, INEC, 2011).

Es importante resaltar la razón ligada a la edad, el costo de la educación, la preferencia por el trabajo o la obligación de hacerlo. En el caso de las mujeres se señala una serie de problemas ligados con tareas tradicionalmente vistas como femeninas como las de reproducción, cuido de personas mayores y embarazo. Lamentablemente, esta información no está desagregada por identidad sociocultural.

En síntesis, el trabajo de menores de edad es una realidad que afecta a un número importante de personas que debieran estar estudiando y capacitándose para su futuro, en el marco de la promesa de la movilidad social ascendente por la vía de la educación. Logro al que la juventud indígena, respecto de todas las demás juventudes, le será más difícil alcanzar.

El mercado laboral, por su parte, no discrimina tanto por etnicidad e identidad cultural como si lo hace por educación. 
doi: http://dx.doi.org/10.15359/ree.20-1.4

URL: http://www.una.ac.cr/educare

CORREO: educare@una.cr

En la siguiente sección nos acercaremos sucintamente a la participación de estas poblaciones en el mercado laboral.

\section{Identidades étnicas y culturales y categoría ocupacional}

En las próximas secciones abordaremos la participación en el mercado laboral de las poblaciones indígenas, afrodescendientes y mestizas desde dos variables: la categoría ocupacional y tipo de ocupación. La categoría ocupacional en la que la persona se inserte, entre otras variables, depende del logro educativo alcanzado. Es importante, entonces, si se quiere alcanzar una buena calidad de vida, o al menos una en la que podamos satisfacer todas las necesidades personales y familiares, conseguir un buen puesto y, concomitantemente, un buen salario.

La categoría de ocupación que prevalece entre las distintas poblaciones indígenas, afrodescendientes y mestizas son las de empleados de empresa privada y el de trabajador por cuenta propia. Hay proporcionalmente pocos patronos. Por otro lado, observando la tabla 4, predominan los asalariados del sector privado y las personas que trabajan por cuenta propia.

Tabla 4

Población indígena, afrodescendiente y mestiza según categoría ocupacional. CensoNacional de Población 2011

\begin{tabular}{lccrc}
\hline \multicolumn{1}{c}{ Categoría ocupacional } & Indígena & Afrodescendiente & Mestiza & \multicolumn{1}{c}{ Total } \\
\hline Patrono(a) (tiene empleados(as) fijos(as) & 1.951 & 1.256 & 92.535 & 107.541 \\
Trabajador(a) por cuenta propia & 11.269 & 3.924 & 287.082 & 333.311 \\
Empleado(a) de empresa privada & 15.037 & 10.026 & 732.121 & 856.870 \\
Empleado(a) del sector público & 4.350 & 3.780 & 223.154 & 251.489 \\
Empleado(a) de casas particulares & 1.813 & 691 & 60.907 & 72.740 \\
Ayudante sin recibir pago & 1.016 & 173 & 14.464 & 16.888 \\
\hline Total & 35.436 & 19.850 & 1.410 .263 & 1.638 .839 \\
\hline
\end{tabular}

Nota: Elaboración propia con base en el X Censo Nacional de Población (INEC, 2011).

En cuanto al tipo de ocupación, las personas se distribuyen en todos los tipos de ocupación, aunque predominan en todas las poblaciones en estudio, las de tipo elemental y la de servicios directos. A este rubro, entre la población indígena le siguen las actividades agrícolas, entre la afrodescendiente, el tipo de empleos profesionales al igual que la mestiza. Después de las ocupaciones científicas, le siguen, en todas las poblaciones, las actividades productivas (artesanía, construcción, mecánica, artes gráficas, etc. (ver tabla 5). 
Tabla 5

Poblaciones indígenas, afrodescendientes y mestizas por tipo de ocupación

\begin{tabular}{|c|c|c|c|c|c|c|}
\hline \multirow[b]{2}{*}{ Ocupación gran grupo } & \multicolumn{2}{|c|}{ Indígenas } & \multicolumn{2}{|c|}{ Afrodescendientes } & \multicolumn{2}{|c|}{ Mestizas } \\
\hline & Fre & $\%$ & Fre & $\%$ & Fre & $\%$ \\
\hline $\begin{array}{l}\text { Nivel directivo, administrativo público y } \\
\text { empresa privada }\end{array}$ & 291 & 0,8 & 271 & 1,4 & 22.905 & 1,6 \\
\hline Nivel profesional, científico e intelectual & 3.179 & 9,0 & 3.007 & 15,1 & 210.760 & 14,9 \\
\hline Nivel técnico y profesional medio & 2.310 & 6,5 & 1.864 & 9,4 & 146.789 & 10,4 \\
\hline Apoyo administrativo & 1.377 & 3,9 & 1.645 & 8,3 & 113.353 & 8,0 \\
\hline $\begin{array}{l}\text { Venta locales y prestación de servicios } \\
\text { directos }\end{array}$ & 6.108 & 17,2 & 4.087 & 20,6 & 281.017 & 19,9 \\
\hline $\begin{array}{l}\text { Agropecuarias, agrícolas y pesqueras } \\
\text { calificadas }\end{array}$ & 5.216 & 14,7 & 682 & 3,4 & 61.117 & 4,3 \\
\hline $\begin{array}{l}\text { Producción artesanal, construcción, } \\
\text { mecánica, artes gráficas y manufacturas } \\
\text { calificadas }\end{array}$ & 3.403 & 9,6 & 2.310 & 11,6 & 160.346 & 11,4 \\
\hline $\begin{array}{l}\text { Operación de instalaciones, máquinas y } \\
\text { ensambladores }\end{array}$ & 1.791 & 5,1 & 1.432 & 7,2 & 124.297 & 8,8 \\
\hline Ocupaciones elementales & 11.761 & 33,2 & 4.552 & 22,9 & 289.679 & 20,5 \\
\hline Total & 35.436 & 100,0 & 19.850 & 100,0 & 1.410 .263 & 100,0 \\
\hline
\end{tabular}

Nota: Elaboración propia con base en el X Censo Nacional de Población (INEC, 2011).

Por otro lado, entre las poblaciones indígenas y afrodescendientes, en términos relativos, hay menos personas ocupadas en condiciones laborales de mayor estatus y remuneración. No obstante, esto es una condición propia del mercado laboral, en donde también entre la población mestiza predominan estas condiciones. No obstante, entre la población de personas mestizas hay también más personas hombres y mujeres ocupando puestos mejor remunerados y mejor estatus social.

A modo de conclusión: El logro de mayores niveles educativos no parece estar ligado con el factor identitario étnico cultural. Lo que se ve descriptivamente es que tanto la cantidad de años de estudios como los niveles educativos alcanzados no dependen particularmente de la pertenencia étnica cultural. Es decir, ser indígena o afrodescendiente no es un factor limitante de logro educativo. Por otro lado, ni la educación que brinda el Estado Costarricense para pueblos indígenas, ni la que se brinda a la comunidad afrocaeribeña hace justicia con la diversidad cultural. El currículo nacional es estándar y uniforme para todo el país. Pero esta discusión es distinta y tiene relación con la 
doi: http://dx.doi.org/10.15359/ree.20-1.4

URL: http://www.una.ac.cr/educare

CORREO: educare@una.cr

contextualización curricular necesaria, aún para los mestizos que viven en distintas zonas del país. Tal como plantea Borge (2012) cuando detalla los logros alcanzados en el territorio bribri cabecar y señala también los pendientes. Estos, centralmente, tienen relación con el hecho de que "....no es posible plantear que exista [una] Educación Indígena en Costa Rica, lo que existe es una educación en Territorios Indígenas" (p. 39). Por su parte, López y Delgado (2013), cuando realizan la síntesis respecto de las desigualdades entre la población afrodescendientes, reconocen que en una alta medida estas obedecen al lugar en donde residen estas personas y no al factor racial.

Por el contrario, el factor de localización y la inserción temprana al mercado laboral sí parecen ser factores limitantes de logro educativo. Sería recomendable indagar el primero de ellos en escalas más pequeñas, tal vez la distrital; y el segundo, observando más de cerca la relación entre logro educativo e ingresos en personas trabajadoras mayores de 15 años, nivel que por bajo que pueda ser parece más atractivo que gastar en educación, la que además ha sido valorada como poco interesante.

No alcanzar logros significativos en educación es, por su parte, una gran limitación para acceder a empleos de calidad, tanto en lo económico como socialmente, porque la educación es vital como factor de minimización de la exclusión laboral. Los empleos menos calificados están peor pagados.

Adicionalmente, una educación baja se transmite de generación en generación, perdiéndose, entre unas y otras, grados diversos de calidad, quedando las generaciones posteriores a merced de los escasos logros educativos, producto de condiciones históricas de transmisión de la pobreza y no de factores transversales como los que se han querido estudiar aquí.

\section{Referencias}

Borge, C. (2012). Costa Rica: Estado de la educación indígena en territorios indígenas (Cuarto Informe del Estado de la Educación. Informe final). San José, Costa Rica. Estado de la Nación.

Bourdieu, P. (1997). Capital cultural, escuela y espacio social (2ª ed.). México: Siglo XXI.

Gacitúa, E.yDavis,S.H.(2000).Introducción.Pobrezayexclusión social en AméricaLatinayElCaribe. En E. Gacitúa, C. Sojo y S. H. Davis (Edits.), Exclusión socialy reducción dela pobreza en América Latina y El Caribe (pp. 13-23). San José, Costa Rica: FLACSO-Banco Mundial. Recuperado de http://info.worldbank.org/etools/docs/library/44254/ExclusionSocialyReducciondePobrezaEnAL.pdf

Gaete, M. (2014). Tasas de logro educativo entre la población afrodescendiente, indígena y mestiza y la revisión de algunos factores de exclusión e inclusión. San José, Costa Rica: Departamento de Estudios e Investigaciones. Ministerio de Educación Pública.

Instituto Nacional de Estadísticas y Censos (INEC). (2011). Encuesta Nacional de Hogares - ENAHO. San José, Costa Rica: Autor. 
López, L. Á. y Delgado, D. (2013). Situación socioeconómica de la población afrodescendiente de Costa Rica según datos del X Censo Nacional de Población y VI de Vivienda 2011. San José, Costa Rica: Programa de Naciones Unidas para el Desarrollo. Recuperado de http://www. cr.undp.org/content/dam/costa rica/docs/undp cr informeafro 2013.pdf

Pérez, J. P. y Mora, M. (2007). La persisitencia de la miseria en Centroamérica. Una mirada desde la exclusión social. FLACSO. San José, Costa Rica: FLACSO. Recuperado dehttp://unpan1.un.org/ intradoc/groups/public/documents/icap/unpan027591.pdf

Perry, G. (2000). Prólogo a las actas del taller sobre pobreza y exclusión social en América Latina. En E. Gacitúa, C. Sojo y S. Davis (Edits.), Exclusión social y reducción de la pobreza en América Latina y El Caribe (pp. 7-10). San José, Costa Rica: FLACSO-Banco Mundial. Recuperado de http://info.worldbank.org/etools/docs/library/44254/ExclusionSocialyReducciondePobrezaEnAL.pdf

Programa de las Naciones Unidas para el Desarrollo (PNUD). (2011). Informe regional sobre desarrollo humano para América Latina y el Caribe. Actuar sobre el futuro: Romper la transmisión intergeneracional de la desigualdad. San José, Costa Rica: Autor.

Programa de las Naciones Unidas para el Desarrollo. (PNUD). (2013). Informenacional sobre desarrollo humano 2013. Aprendiendo a vivir juntos: Convivencia y desarrollo humano en Costa Rica.. San José, Costa Rica: Autor. Recuperado de http://hdr.undp.org/sites/default/files/costa rica nhdr 2013.pdf

Sauma, P.y Trejos, J. D. (2014). Reducir la pobreza en Costa Rica es posible. Propuestas para la acción. Programa de Naciones Unidas para el Desarrollo. San José, Costa Rica: PNUD. Recuperado de http://www.cr.undp.org/content/dam/costa rica/docs/undp cr reducirpobreza 2014.pdf

Sojo, C. (2000). Dinámica sociopolítica y cultural de la exclusión social. En E. Gacitúa, C. Sojo y S. H. Davis (Edits.), Exclusión social y reducción de la pobreza en América Latina y El Caribe (pp. 51-89). San José, Costa Rica: FLACSO-Banco Mundial. Recuperado de http://info. worldbank.org/etools/docs/library/44254/ExclusionSocialyReducciondePobrezaEnAL.pdf

UNICEF Costa Rica. (2010). Así vivimos los pueblos indígenas. Diagnóstico niñez y adolescencia indígena. San José, Costa Rica: UNICEF-MNI. Recuperado de http://www.unicef.org/costarica/ docs/cr pub Asi vivimos los pueblos indigenas.pdf

\section{Cómo citar este artículo en APA:}

Gaete-Astica, M. (Enero-abril, 2016). Tasas de logro educativo en las poblaciones afrodescendiente, indígena y mestiza: Revisión de algunos factores de exclusión. Revista Electrónica Educare, 20(1), 1-21. doi: http:// dx.doi.org/10.15359/ree.20-1.4

Nota: Para citar este artículo en otros sistemas puede consultar el hipervínculo "Como citar el artículo" en la barra derecha de nuestro sitio web: http://www.revistas.una.ac.cr/index.php/EDUCARE/index 